\title{
Satellite operators as group actions on knot concordance
}

\author{
CHRISTOPHER W DAVIS \\ ARUNIMA RAY
}

\begin{abstract}
Any knot in a solid torus, called a pattern, induces a function, called a satellite operator, on concordance classes of knots in $S^{3}$ via the satellite construction. We introduce a generalization of patterns that form a group (unlike traditional patterns), modulo a generalization of concordance. Generalized patterns induce functions, called generalized satellite operators, on concordance classes of knots in homology spheres; using this we recover the recent result of Cochran and the authors that patterns with strong winding number \pm 1 induce injective satellite operators on topological concordance classes of knots, as well as smooth concordance classes of knots modulo the smooth 4-dimensional Poincaré conjecture. We also obtain a characterization of patterns inducing surjective satellite operators, as well as a sufficient condition for a generalized pattern to have an inverse. As a consequence, we are able to construct infinitely many nontrivial patterns $P$ such that there is a pattern $\bar{P}$ for which $\bar{P}(P(K))$ is concordant to $K$ (topologically as well as smoothly in a potentially exotic $\left.S^{3} \times[0,1]\right)$ for all knots $K$; we show that these patterns are distinct from all connected-sum patterns, even up to concordance, and that they induce bijective satellite operators on topological concordance classes of knots, as well as smooth concordance classes of knots modulo the smooth 4-dimensional Poincaré conjecture.
\end{abstract}

\section{$57 \mathrm{M} 25$}

\section{Introduction}

The satellite construction is a classical and well-studied family of functions on the set of knots in $S^{3}$. Briefly, the satellite construction involves a pattern, $P$, ie a knot in a solid torus $V=S^{1} \times D^{2}$, and a knot $K$ in $S^{3}$, called the companion; the satellite knot $P(K)$ is the image of the knot $P$ when the solid torus $V$ is tied into the knot $K$; see Figure 1 and Section 2.

We will consider four different equivalence relations on knots, with corresponding sets of equivalence classes $\mathcal{C}, \mathcal{C}_{\text {top }}, \mathcal{C}_{\text {ex }}$, and $\mathcal{C}_{R}$, where $R$ is any localization of $\mathbb{Z}$. Here $\mathcal{C}$ denotes the smooth knot concordance group, consisting of knots up to smooth concordance; we recall that two knots $K_{0} \hookrightarrow S^{3} \times\{0\}$ and $K_{1} \hookrightarrow S^{3} \times\{1\}$ are smoothly 


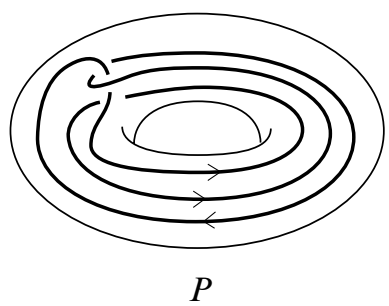

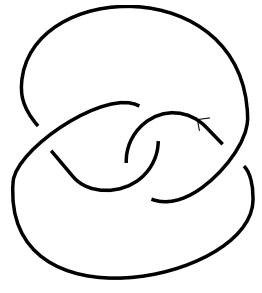

$K$

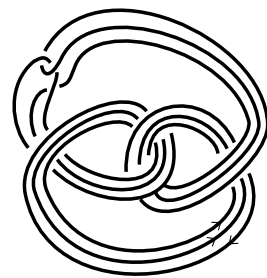

$P(K)$

Figure 1: The satellite construction on knots in $S^{3}$.

concordant if they cobound a smooth, properly embedded annulus in $S^{3} \times[0,1]$ with its usual smooth structure. Similarly, $K_{0} \hookrightarrow S^{3} \times\{0\}$ and $K_{1} \hookrightarrow S^{3} \times\{1\}$ are topologically concordant if they cobound a locally flat, properly embedded annulus in a topological manifold homeomorphic to $S^{3} \times[0,1]$; knots modulo topological concordance form the topological concordance group, denoted $\mathcal{C}_{\text {top }}$. As a transition of sorts between $\mathcal{C}$ and $\mathcal{C}_{\text {top }}$, we have the exotic concordance group $\mathcal{C}_{\text {ex }}$, consisting of knots up to exotic concordance, where $K_{0} \hookrightarrow S^{3} \times\{0\}$ and $K_{1} \hookrightarrow S^{3} \times\{1\}$ are exotically concordant if they cobound a smooth, properly embedded annulus in a smooth manifold homeomorphic, but not necessarily diffeomorphic, to $S^{3} \times[0,1]$; ie $\mathcal{C}_{\text {ex }}$ consists of knots up to concordance in a potentially exotic $S^{3} \times[0,1]$. For a localization $R$ of $\mathbb{Z}$, the knots $K_{0} \hookrightarrow S^{3} \times\{0\}$ and $K_{1} \hookrightarrow S^{3} \times\{1\}$ are $R$-concordant if they cobound a smooth, properly embedded annulus in a smooth $R$-homology cobordism from $S^{3} \times\{0\}$ to $S^{3} \times\{1\} ; \mathcal{C}_{R}$ denotes the group of knots up to $R$-concordance. If the smooth 4-dimensional Poincaré conjecture is true then $\mathcal{C}_{\mathrm{ex}}=\mathcal{C}$ [5, Proposition 3.2]. In fact, for odd $n$, it is unknown whether $\mathcal{C}_{\mathbb{Z}[1 / n]}=\mathcal{C} .\left(\mathcal{C}_{\mathbb{Z}[1 / 2]}\right.$ is distinct from $\mathcal{C}$, since the figure-eight knot is slice in a $\mathbb{Z}\left[\frac{1}{2}\right]$-homology ball, but not slice.)

The satellite construction is well-defined on concordance classes of each of the types mentioned above; that is, any pattern $P$ gives a function, a satellite operator (which we will denote by the same letter),

$$
\begin{aligned}
P: \mathcal{C}_{*} & \rightarrow \mathcal{C}_{*}, \\
{[K] } & \mapsto[P(K)],
\end{aligned}
$$

where $* \in\{\varnothing$, ex, top, $R\}$ for any localization $R$ of $\mathbb{Z}$, and $[\cdot]$ denotes the relevant concordance class. This fact has been used to construct knots that yield distinct concordance classes but which cannot be distinguished between by many classical invariants. Examples of this philosophy in action can be found in [8; 7]. In [6], winding number one patterns are used to construct nonconcordant knots which have homology cobordant zero surgery manifolds. In the more general context of 3-and 4-manifold topology, the satellite construction was used in [10] to modify a 3-manifold while 
fixing its homology type. Winding number one patterns, which are of particular interest in this paper, are related to Mazur 4-manifolds [3] and Akbulut corks [2]. In [4], it was shown that changing the attaching curve of a 2-handle in a handlebody description of a 4-manifold by a winding number one satellite construction can change the (relative) diffeomorphism type while fixing the homeomorphism type.

As a result, there has been considerable interest in understanding satellite operators on $\mathcal{C}$. For example, it is a famous conjecture that the Whitehead double of a knot $K$ is smoothly slice if and only if $K$ is smoothly slice [13, Problem 1.38]. This question might be generalized to ask if satellite operators are injective on smooth concordance classes; that is, if $P$ is a pattern/satellite operator, does $P(K)=P(J)$ imply $K=J$ in smooth concordance? A survey of such work on the Whitehead doubling operator may be found in [11]. In [7], several "robust doubling operators" were introduced, and some evidence was provided for their injectivity. This is the current state of knowledge in the winding number zero case. For satellite operators induced by patterns with nonzero winding numbers, there has been more success as seen in the following recent theorem.

Theorem 1.1 ([5, Theorem 5.1]; see Corollary 2.16) Suppose $P$ is a pattern with nonzero winding number $n$. Then

(a) $\quad P: \mathcal{C}_{\mathbb{Z}[1 / n]} \rightarrow \mathcal{C}_{\mathbb{Z}[1 / n]}$ is injective.

Suppose that $P$ is a pattern with strong winding number \pm 1 . Then

(b) $P: \mathcal{C}_{\text {ex }} \rightarrow \mathcal{C}_{\text {ex }}$ is injective,

(c) $P: \mathcal{C}_{\text {top }} \rightarrow \mathcal{C}_{\text {top }}$ is injective, and

(d) if the smooth 4-dimensional Poincaré conjecture holds, $P: \mathcal{C} \rightarrow \mathcal{C}$ is injective.

See Section 2 for a definition of strong winding number; for the moment it suffices to know that patterns with strong winding number \pm 1 are plentiful. The above theorem has a number of useful corollaries (see [5]) and gives us a valuable tool in studying satellite knots. We will see in Section 2 that it will follow easily from the main theorem of this paper.

Let $\mathcal{S}$ denote the set of all patterns. $\mathcal{S}$ has a monoid structure with respect to which the usual satellite construction is a monoid action; see Section 2. The main technical result of this paper shows that the classical satellite construction is, in fact, a restriction of a natural group action. Specifically, we show that patterns form a submonoid of the group of homology cobordism classes of homology cylinders, which was introduced by Levine in [15], and we show that this group has a natural action on concordance classes of knots in homology spheres, which is compatible with the classical satellite construction. In other words, we prove a theorem of the following type. 
Main theorem (See Section 2 for the precise statement) Let $* \in\{$ ex, top, $R\}$ for a localization $R$

of $\mathbb{Z}$. For each $\mathcal{C}_{*}$, there exist a particular submonoid $\mathcal{S}_{*}$ of $\mathcal{S}$, an enlargement

$$
\Psi: \mathcal{C}_{*} \hookrightarrow \widehat{\mathcal{C}}_{*},
$$

and a monoid morphism,

$$
E: \mathcal{S}_{*} \rightarrow \widehat{\mathcal{S}}_{*},
$$

where $\widehat{\mathcal{S}}_{*}$ is a group which acts on $\widehat{\mathcal{C}}_{*}$, such that the following diagram commutes for all $P \in \mathcal{S}_{*}$ :

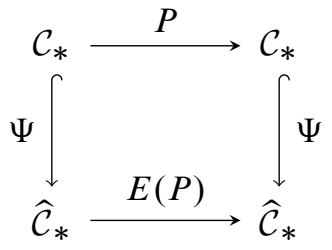

In the above, $\mathcal{S}_{*}$ contains all strong winding number \pm 1 patterns for $*=$ ex or top, and all winding number $\pm n$ patterns when $*=\mathbb{Z}\left[\frac{1}{n}\right] ; \widehat{\mathcal{C}}_{*}$ consists of knots in homology spheres up to concordance in the category $*$; and $\widehat{\mathcal{S}}_{*}$ is a group of homology cobordism classes of homology cylinders. For a pattern $P \subseteq V=S^{1} \times D^{2}$, we denote by $E(P)$ the exterior of a regular neighborhood of $P$ in $V$, as well as the function on $\widehat{\mathcal{C}}_{*}$ induced by it. See Section 2 for the precise definitions as well as the proof of the main theorem.

Since $E(P)$ is an element of a group acting on $\widehat{\mathcal{C}}_{*}, E(P): \widehat{\mathcal{C}}_{*} \rightarrow \widehat{\mathcal{C}}_{*}$ must be a bijection. This observation yields Theorem 1.1 as a corollary (see Corollary 2.16), via an elementary diagram chase.

Moreover, considering the satellite construction as a restriction of a group action provides a novel approach to the problem of finding nontrivial surjective satellite operators on $\mathcal{C}_{*}$. While it is elementary to show that patterns with winding number other than \pm 1 cannot induce surjections on knot concordance (see Proposition 3.1), very little is known in the case of patterns of winding number \pm 1 . For instance, the conjecture of Akbulut [13, Problem 1.45] that there exists a winding number one pattern $P$ such that $P(K)$ is not slice for any knot $K$ (that is, the unknot is not in the image of the satellite operator) remains open.

For $* \in\{$ ex, top, $R\}$ and $P$ in the monoid $\mathcal{S}_{*}$, we have shown that $E(P)$ is an element of a group and, therefore, has a well-defined inverse $E(P)^{-1}$. It is of independent 
interest to ask whether, for a given pattern $P$, the homology cylinder $E(P)^{-1}$ corresponds to a pattern, that is, if there exists some $\bar{P} \in \mathcal{S}$ such that $E(P)^{-1}=E(\bar{P})$ in $\mathcal{S}_{*}$ (we say that $\bar{P}$ is an inverse for $P$ ). In fact, if $P$ has an inverse $\bar{P}$, we may infer that the induced satellite operator $P$ is a bijection on $\mathcal{C}_{*}$; see Corollary 3.7. In Section 3, we give a sufficient condition for a pattern to have an inverse, which allows us to construct a family of patterns that induce bijective satellite operators on $\mathcal{C}_{*}$, as follows. Of course, it is easy to see that connected-sum patterns (of the form shown in Figure 5) give bijective functions on $\mathcal{C}_{*}$ for each $* \in\{$ ex, top, $R\}$. Consequently, when seeking examples of patterns inducing bijective satellite operators, one should make sure that one avoids patterns that are equivalent to connected sum patterns.

Theorem 3.4 Let $P \subseteq V=S^{1} \times D^{2}$ be a pattern with winding number \pm 1 . If the meridian of $P$ is in the normal subgroup of $\pi_{1}(E(P))$ generated by the meridian of $V$, then $P$ has strong winding number \pm 1 , and there exists another strong winding number \pm 1 pattern $\bar{P}$ such that $E(P)^{-1}=E(\bar{P})$ as homology cylinders.

Corollary 3.9 For each $m \geq 0$, the pattern $P_{m} \subseteq V\left(P_{m}\right)=S^{1} \times D^{2}$ shown in Figure 2 induces a bijective map $P_{m}: \mathcal{C}_{*} \rightarrow \mathcal{C}_{*}$ for $* \in\{$ ex, top, $\mathbb{Z}\}$. Moreover, each $P_{m}$ is distinct from all connected-sum patterns.

In our final application of the main theorem, we draw a connection between the surjectivity of satellite operators (specifically Akbulut's conjecture [13, Problem 1.45] mentioned above) and a question of Matsumoto [13, Problem 1.30] asking if every knot in a 3-manifold homology cobordant to $S^{3}$ is concordant, in a homology cobordism to $S^{3}$, to a knot in $S^{3}$. That is, we show the following result.

Proposition 4.3 Let $* \in\{$ ex, top $\}$. If there exists a pattern with strong winding number \pm 1 such that the induced function $P: \mathcal{C}_{*} \rightarrow \mathcal{C}_{*}$ is not surjective, then there exists a

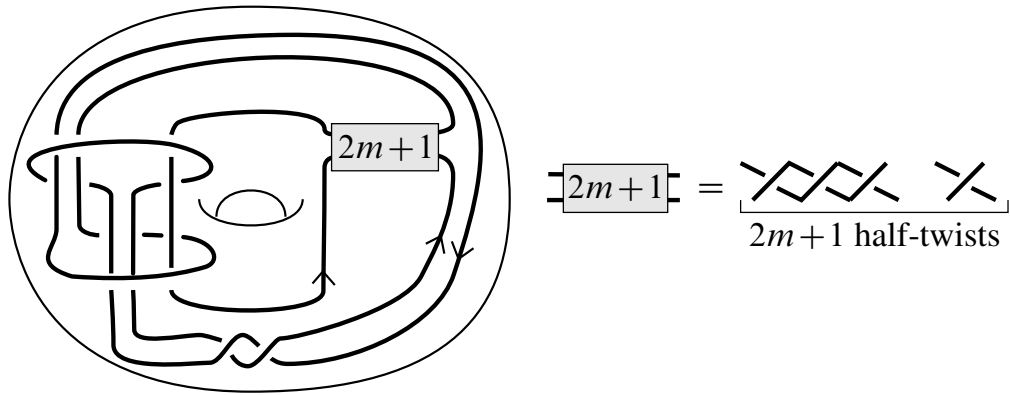

Figure 2: $\left\{P_{m}\right\}_{m \geq 0}$, a class of patterns yielding bijective satellite operators (see Corollary 3.9). 
knot in a 3-manifold $M$ homology cobordant to $S^{3}$ that is not concordant, in the category $*$, to any knot in $S^{3}$ in any $*$-homology cobordism from $M$ to $S^{3}$.

Outline In Section 2, we give the relevant background and prove the main theorem. Section 3 addresses surjectivity of satellite operators. In Section 4, we prove Proposition 4.3.

Remark Shortly after a preprint of this paper was circulated, Adam Levine proved the existence of nonsurjective satellite operators; see [14].

\section{Background and proof of the main theorem}

\subsection{Patterns and satellite operators}

For a pattern $P \subseteq V=S^{1} \times D^{2}$, let $E(P)$ denote the complement of a regular neighborhood of $P$ in $V$. There are four important (oriented) curves on the boundary of $E(P)$ :

(1) $m(P)$, the meridian of the pattern,

(2) $\ell(P)$, the longitude of the pattern,

(3) $m(V)=m(V(P))=1 \times \partial D^{2}$, the meridian of the solid torus, and

(4) $\ell(V)=\ell(V(P))=S^{1} \times 1$, the longitude of the solid torus.

Here, $\ell(P)$ is the pushoff (unique up to isotopy) of $P$ in $V$, which is homologous in $E(P)$ to a multiple of $\ell(V)$. We say that $P$ has winding number $w=w(P) \in \mathbb{Z}$ if $\ell(P)$ is homologous to $w \cdot \ell(V)$. Consistent with this definition, on the torus $T=S^{1} \times S^{1}$, we will call the curve $\ell=S^{1} \times\{1\}$ the longitude of $T$, and the curve $m=\{1\} \times S^{1}$ the meridian of $T$. Let $\mathcal{S}$ denote the set of all patterns up to isotopy.

Remark 2.1 Given a pattern $P \subseteq V$ one gets a knot $\widetilde{P} \subseteq S^{3}$ by adding a 2-handle to $V$ along $\ell(V)$, followed by a 3-handle along the resulting 2 -sphere boundary. Let $\eta$ be the image of $m(V)$ after this handle addition. It is straightforward to see that the map $P \mapsto(\widetilde{P}, \eta)$ gives a bijection from $\mathcal{S}$ to the set of (ordered, oriented) 2-component links in $S^{3}$ whose second component is unknotted, since, given such a link, we can remove a tubular neighborhood of the second component to get a knot (the first component) in a solid torus, namely a pattern. The winding number of $P$ is the same as the linking number between $\widetilde{P}$ and $\eta$, and $E(P)$ is the complement of the link $(\widetilde{P}, \eta)$ in $S^{3}$; see Figure 3 . 


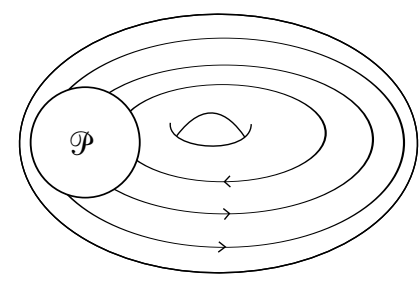

(a)

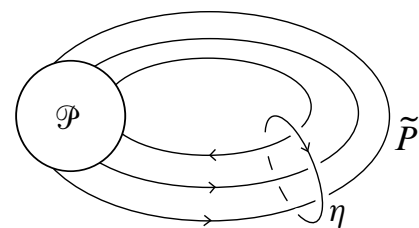

(b)

Figure 3: (a) A schematic picture of a pattern $P$. The circle containing $\mathscr{P}$ denotes a tangle. (b) The 2-component link corresponding to the pattern $P$. The circle containing $\mathscr{P}$ denotes the same tangle as in the previous panel.

The set $\mathcal{S}$ has a natural monoid structure. Given two patterns $P \subseteq V(P)$ and $Q \subseteq V(Q)$, where $V(P)$ and $V(Q)$ are standard solid tori, we construct the composed pattern $P \star Q$ as follows. Glue $E(Q)$ and $V(P)$ together by identifying $\partial E(Q)$ with $\partial V(P)$ via $m(Q) \sim m(V(P))$ and $\ell(Q) \sim \ell(V(P))$. The product $P \star Q$ is the image of $P$ after this identification, and the resulting manifold is still a solid torus $V(P \star Q)$; see Figure 4. The operation $\star$ is clearly associative, ie $P \star(Q \star S)=(P \star Q) \star S$, and the monoid identity is given by the core of the solid torus, namely the trivial pattern.

Patterns act on knots in $S^{3}$ as we described in Figure 1. To obtain $P(K)$ from a pattern $P \subseteq V$ and a knot $K \subseteq S^{3}$, start with the knot complement $S^{3}-K$. The toral boundary contains the oriented curves $\ell(K)$, the longitude of $K$, and $m(K)$, the meridian of $K$. Glue in $V(P)$ by identifying $\ell(V) \sim \ell(K)$ and $m(V) \sim m(K)$. The resulting 3-manifold is $S^{3}$, and the image of $P$ is the satellite knot $P(K)$. For further details, see [18, page 111].

Let $\mathcal{K}$ denote the set of knots in $S^{3}$ modulo isotopy. For patterns $P$ and $Q$ and a knot $K$, we easily see that $(P \star Q)(K)=P(Q(K))$. Therefore, we have a monoid homomorphism $\mathcal{S} \rightarrow \operatorname{Maps}(\mathcal{K}, \mathcal{K})$, that is, a monoid action on the set of isotopy classes of knots in $S^{3}$. The following proposition shows that $\mathcal{S}$ is far from being a group under the operation $\star$.
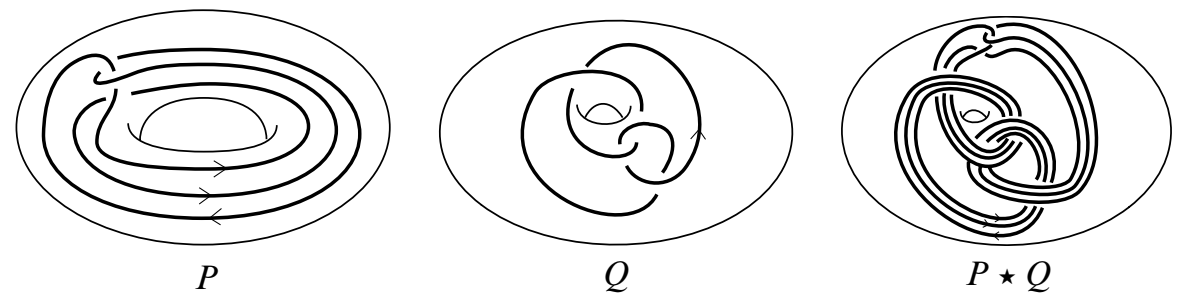

Figure 4: The monoid operation on patterns. 
Proposition 2.2 The only element of $\mathcal{S}$ that has an inverse under the operation $\star$ is the trivial pattern given by the core of the solid torus.

Proof We see this using the notion of bridge index $\mathrm{b}(K)$ of a knot $K$; see [18, p. 114]. Suppose $P \subseteq V$ has an inverse denoted $P^{-1}$. For any satellite knot $P(K)$, we know from [19] that $\mathrm{b}(P(K)) \geq n \cdot \mathrm{b}(K)$, where $n$ is the geometric winding number of $P$, ie the minimal (unsigned) number of intersections between $\ell(P)$ and a meridional disk of $V$. Therefore, for any pattern $P$ and knot $K, \mathrm{~b}(P(K)) \geq \mathrm{b}(K)$, and so

$$
\mathrm{b}(K)=\mathrm{b}\left(\left(P^{-1} \star P\right)(K)\right)=\mathrm{b}\left(P^{-1}(P(K))\right) \geq \mathrm{b}(P(K)) \geq \mathrm{b}(K) .
$$

Thus, $\mathrm{b}(P(K))=\mathrm{b}(K)$, and $P$ has geometric winding number one. This implies that $P$ is a connected-sum pattern, ie $P=Q_{J}$ for some knot $J$ as shown in Figure 5. However, the connected-sum operation with a nontrivial knot cannot have an inverse due to the additivity of genus. Therefore, $P=Q_{U}$, where $U$ is the unknot. This completes the proof, since $Q_{U}$ is the trivial pattern.

The following submonoids of $\mathcal{S}$ will be of particular interest in this paper.

Definition 2.3 Let $P$ be a pattern and $\mathbb{Z} \subseteq R \subseteq \mathbb{Q}$ a localization of $\mathbb{Z}$.

(a) $\quad P$ is said to lie in $\mathcal{S}_{R}$ if $w(P)$ is invertible in $R$, that is, $1 / w(P) \in R$.

(b) $P$ is said to lie in $\mathcal{S}_{\text {str }}$ (and have strong winding number \pm 1 ) if $w(P)=$ \pm 1 and each of the sets $\{m(V), \ell(V)\}$ and $\{m(P), \ell(P)\}$ normally generates $\pi_{1}(E(P))$.

Recall from Remark 2.1 that one can obtain a knot $\widetilde{P}$ from a pattern $P \subseteq V=S^{1} \times D^{2}$ by adding a 3-dimensional 2-handle to $V$ along $\ell(V)$ and then a 3-dimensional 3-handle along the resulting 2-sphere boundary (in fact, $\widetilde{P}=P(U)$, where $U$ is the unknot). In [5], a pattern $P$ was said to have strong winding number \pm 1 if $w(P)= \pm 1$ and $m(V)$ normally generates $\pi_{1}\left(S^{3}-\tilde{P}\right)$. Our definition is equivalent to the definition in [5] as follows.

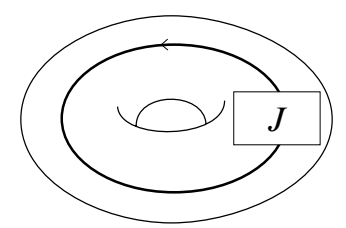

Figure 5: The connected-sum pattern $Q_{J}$ corresponding to the knot $J$. A strand going up through the box marked $J$ has the knot type of $J$. 
Proposition 2.4 For a pattern $P \subseteq V=S^{1} \times D^{2}$ with winding number \pm 1 , we have that $m(V)$ normally generates $\pi_{1}\left(S^{3}-\widetilde{P}\right)$ if and only if each of the sets $\{m(V), \ell(V)\}$ and $\{m(P), \ell(P)\}$ normally generates $\pi_{1}(E(P))$.

Proof From the definition of $\widetilde{P}$, it is clear that $\pi_{1}\left(S^{3}-\widetilde{P}\right)=\pi_{1}(E(P)) /\langle\langle\ell(V)\rangle$, where $\langle\langle\ell(V)\rangle\rangle$ is the normal subgroup generated by $\ell(V)$ in $\pi_{1}(E(P))$. The backward direction follows immediately, since if $\{m(V), \ell(V)\}$ normally generates $\pi_{1}(E(P))$, the group $\pi_{1}\left(S^{3}-\widetilde{P}\right)=\pi_{1}(E(P)) /\langle\langle\ell(V)\rangle\rangle$ must be normally generated by $m(V)$. For the forward direction, note that if we assume that $m(V)$ normally generates $\pi_{1}\left(S^{3}-\widetilde{P}\right)=\pi_{1}(E(P)) /\left\langle\langle\ell(V)\rangle\right.$, then $\{m(V), \ell(V)\}$ normally generates $\pi_{1}(E(P))$. In order to see that $\{m(P), \ell(P)\}$ also normally generates $\pi_{1}(E(P))$, notice that $V$ is obtained from $E(P)$ by adding a 2-handle along $m(P)$ (followed by a 3-handle) so that

$$
\left.\mathbb{Z} \cong \pi_{1}(V)=\pi_{1}(E(P)) /\langle m(P)\rangle\right\rangle .
$$

Since $P$ has winding number \pm 1 , we know $\pi_{1}(V)$ is generated by $\ell(P)$, which is homotopic in $V$ to $\ell(V)$. Thus, $\{m(P), \ell(P)\}$ normally generates $\pi_{1}(E(P))$.

\subsection{Homology cobordism classes of homology cylinders}

In [15], Levine defined the group of integral homology cylinders over a surface, with the goal of producing an enlargement of the mapping class group. For completeness, and since we require slight variants and generalizations, we recall the definitions below.

Definition 2.5 [15] Let $T=S^{1} \times S^{1}$ be the torus and $R$ a localization of $\mathbb{Z}$. An $R$-homology cylinder on $T$, or an $R$-cylinder, is a triple $\left(M, i_{+}, i_{-}\right)$where

- $\quad M$ is an oriented, compact, connected 3-manifold;

- $i_{\epsilon}: T \rightarrow \partial M$ is an embedding, for $\epsilon= \pm 1$, with $\partial M=i_{+}(T) \sqcup i_{-}(T)$;

- $i_{+}$is orientation-preserving and $i_{-}$is orientation-reversing; and

- $\left(i_{\epsilon}\right)_{*}: H_{*}(T ; R) \rightarrow H_{*}(M ; R)$ is an isomorphism.

For $R$-homology cylinders $\left(M, i_{+}, i_{-}\right)$and $\left(N, j_{+}, j_{-}\right)$, we say that $\left(M, i_{+}, i_{-}\right)=$ $\left(N, j_{+}, j_{-}\right)$if there is a homeomorphism $\phi: M \rightarrow N$ such that $\phi \circ i_{\epsilon}=j_{\epsilon}$ for $\epsilon \in\{ \pm 1\}$.

A $\mathbb{Z}$-cylinder $\left(M, i_{+}, i_{-}\right)$is called a strong cylinder if $\pi_{1}(M)$ is normally generated by each of $\operatorname{Im}\left(i_{+}\right)_{*}$ and $\operatorname{Im}\left(i_{-}\right)_{*}$. Let $H_{R}$ denote the set of all $R$-cylinders and $H_{\text {str }}$ denote the set of all strong cylinders. 
For $* \in\{$ str, $R\}$, there is a monoid operation on $H_{*}$ given by stacking:

$$
\left(M, i_{+}, i_{-}\right) \star\left(N, j_{+}, j_{-}\right)=\left(M \sqcup N / i_{+}(x) \sim j_{-}(x), \forall x \in T, j_{+}, i_{-}\right) .
$$

The identity element with respect to $\star$ is given by $(T \times[0,1], \mathrm{id} \times\{1\}, \mathrm{id} \times\{0\})$.

Definition 2.6 [15] Two $R$-cylinders $\left(M, i_{+}, i_{-}\right)$and $\left(N, j_{+}, j_{-}\right)$are said to be $R$-cobordant if there is a smooth $4-$ manifold $W$ with

$$
\partial W=M \sqcup-N / i_{+}(x) \sim j_{+}(x), i_{-}(x) \sim j_{-}(x), \forall x \in T
$$

such that $H_{*}(M ; R) \rightarrow H_{*}(W ; R)$ and $H_{*}(N ; R) \rightarrow H_{*}(W ; R)$ are isomorphisms. This is equivalent to requiring that the compositions

$$
\begin{gathered}
H_{*}(T ; R) \stackrel{\left(i_{\epsilon}\right)_{*}}{\longrightarrow} H_{*}(M ; R) \longrightarrow H_{*}(W ; R), \\
H_{*}(T ; R) \stackrel{\left(j_{\epsilon}\right)_{*}}{\longrightarrow} H_{*}(N ; R) \longrightarrow H_{*}(W ; R)
\end{gathered}
$$

are isomorphisms for each $\epsilon \in\{ \pm 1\}$. Such a $W$ is called an $R$-cobordism. $\mathcal{H}_{R}$ denotes the set of all $R$-cobordism classes of $R$-cylinders.

Two strong cylinders $\left(M, i_{+}, i_{-}\right)$and $\left(N, j_{+}, j_{-}\right)$are said to be strongly cobordant if there exists a $\mathbb{Z}$-cobordism $W$ between $M$ and $N$ such that $\pi_{1}(W)$ is normally generated by each of $\pi_{1}(M)$ and $\pi_{1}(N)$. Such a $W$ is called a strong cobordism. This is equivalent to requiring that the images of

$$
\begin{aligned}
& \pi_{1}(T) \stackrel{\left(i_{\epsilon}\right)_{*}}{\longrightarrow} \pi_{1}(M) \longrightarrow \pi_{1}(W), \\
& \pi_{1}(T) \stackrel{\left(j_{\epsilon}\right)_{*}}{\longrightarrow} \pi_{1}(N) \longrightarrow \pi_{1}(W)
\end{aligned}
$$

(individually) normally generate $\pi_{1}(W)$ for each $\epsilon \in\{ \pm 1\}$. We let $\mathcal{H}_{\mathrm{ex}}$ denote the set of all strong cobordism classes of strong cylinders.

In the latter definition, if the manifold $W$ is not required to be smooth, we say $\left(M, i_{+}, i_{-}\right)$and $\left(N, j_{+}, j_{-}\right)$are strongly topologically cobordant. $\mathcal{H}_{\text {top }}$ denotes the set of strong topological cobordism classes of strong cylinders.

In [15], Levine proves that the binary operation $\star$ on $H_{\mathbb{Z}}$ is well-defined on $\mathcal{H}_{\mathbb{Z}}$ and endows $\mathcal{H}_{\mathbb{Z}}$ with the structure of a group. Indeed, $N \times[0,1]$ can be seen to be a cobordism between $\left(N, i_{+}, i_{-}\right) \star\left(-N, i_{-}, i_{+}\right)$, where $-N$ denotes the orientation reverse of $N$, and the identity element $(T \times[0,1], \mathrm{id} \times\{0\}, \mathrm{id} \times\{1\})$. Thus, the inverse of $\left(N, i_{+}, i_{-}\right)$in $\mathcal{H}_{\mathbb{Z}}$ is $\left(-N, i_{-}, i_{+}\right)$. Since $N \times[0,1]$ is a smooth $R$-cobordism when $N$ is an $R$-cylinder, $\mathcal{H}_{R}$ is a group for all localizations $R$ of $\mathbb{Z}$. Similarly, if $N$ is a strong cylinder, it is easy to see that $N \times[0,1]$ is a smooth and topological strong cobordism. Thus, $\mathcal{H}_{\text {ex }}$ and $\mathcal{H}_{\text {top }}$ are also groups. 


\subsection{Patterns produce homology cylinders}

For any pattern $P \in \mathcal{S}_{R}$, the exterior of $P$ in the solid torus, $E(P)$, can be seen to be an $R$-homology cylinder in a natural way. Let $i_{+}$be the identification $S^{1} \times S^{1} \rightarrow$ $\partial V$ sending $m \mapsto m(V)$ and $\ell \mapsto \ell(V)$. Similarly, let $i_{-}$be the identification of the boundary of a tubular neighborhood of $P$ with $S^{1} \times S^{1}$ that sends $\ell \mapsto \ell(P)$ and $m \mapsto m(P)$. A Mayer-Vietoris argument easily reveals that $\left(E(P), i_{+}, i_{-}\right)$is an $R$-cylinder. It follows immediately from our definitions that if $P \in \mathcal{S}_{\text {str }}$, then $\left(E(P), i_{+}, i_{-}\right) \in H_{\text {str }}$. Henceforth, we will often abuse notation by letting $E(P)$ denote the $*$-cylinder $\left(E(P), i_{+}, i_{-}\right)$, where $* \in\{$ str, $R\}$. For each value of $*$, we have a map

$$
E: \mathcal{S}_{*} \rightarrow H_{*}, \quad P \mapsto E(P),
$$

which is easily seen to be a monoid homomorphism.

Note that if $P$ is a pattern with winding number $w \neq 0$, then in $E(P)$, the curve $\ell(P)$ is homologous to $w \cdot \ell(V)$, and the curve $m(P)$ is homologous to $(1 / w) \cdot m(V)$. Thus, with respect to the basis $\{\ell, m\}$ for $H_{1}\left(S^{1} \times S^{1}\right)$, the composition $\left(\left(i_{+}\right)_{*}^{-1} \circ\left(i_{-}\right)_{*}\right)$ is given by the matrix $\left[\begin{array}{cc}w & 0 \\ 0 & 1 / w\end{array}\right]$. This motivates the following definition.

Definition 2.7 Let $R$ be a localization of $\mathbb{Z}$. We define $\hat{\mathcal{S}}_{R}^{0} \subseteq H_{R}$ to be the submonoid of all $R$-cylinders $\left(M, i_{+}, i_{-}\right)$for which the map $\left(i_{+}\right)_{*}^{-1} \circ\left(i_{-}\right)_{*}: H_{1}(T ; R) \rightarrow$ $H_{1}(T ; R)$ has determinant one and is diagonal with respect to the basis $\{\ell, m\}$. Similarly, $\hat{\mathcal{S}}_{\text {str }}^{0} \subseteq H_{\text {str }}$ is the submonoid of all strong cylinders $\left(M, i_{+}, i_{-}\right)$for which the map $\left(i_{+}\right)_{*}^{-1} \circ\left(i_{-}\right)_{*}: H_{1}(T ; \mathbb{Z}) \rightarrow H_{1}(T ; \mathbb{Z})$ is \pm id. Elements of $\hat{\mathcal{S}}_{*}^{0}$ will be called generalized $*$-patterns, for $* \in\{$ str, $R\}$.

As a result of our previous discussion, we see the following result.

Proposition 2.8 For each $* \in\{$ str, $R\}$ there is a monoid homomorphism

$$
E: \mathcal{S}_{*} \rightarrow \hat{\mathcal{S}}_{*}^{0} \text {. }
$$

Moreover, the submonoids $\hat{\mathcal{S}}_{*}^{0}$ of $H_{*}$ are closed under the map sending a $*$-cylinder to its inverse in $\mathcal{H}_{*}$, namely $\left(N, i_{+}, i_{-}\right) \mapsto\left(-N, i_{-}, i_{+}\right)$. Therefore, we define

$$
\begin{aligned}
\hat{\mathcal{S}}_{\text {ex }} & :=\hat{\mathcal{S}}_{\text {str }}^{0} / \text { strong cobordism, } \\
\hat{\mathcal{S}}_{\text {top }} & :=\widehat{\mathcal{S}}_{\text {str }}^{0} / \text { strong topological cobordism, } \\
\hat{\mathcal{S}}_{R} & :=\widehat{\mathcal{S}}_{R}^{0} / R \text {-cobordism }
\end{aligned}
$$

and see that $\hat{\mathcal{S}}_{\text {ex }}, \hat{\mathcal{S}}_{\text {top }}$, and $\hat{\mathcal{S}}_{R}$ are subgroups of $\mathcal{H}_{\text {ex }}, \mathcal{H}_{\text {top }}$, and $\mathcal{H}_{R}$, respectively. 
From the above, using the monoid morphisms $E$ from Proposition 2.8, we obtain the following proposition.

Proposition 2.9 There are monoid homomorphisms

$$
E: \mathcal{S}_{R} \rightarrow \widehat{\mathcal{S}}_{R}, \quad E: \mathcal{S}_{\text {str }} \rightarrow \widehat{\mathcal{S}}_{\text {ex }}, \quad \text { and } \quad \mathcal{S}_{\text {str }} \rightarrow \widehat{\mathcal{S}}_{\text {top }}
$$

\subsection{Generalizations of knot concordance}

Definition 2.10 Let $K$ and $J$ be knots in the $\mathbb{Z}$-homology spheres $X$ and $Y$, respectively. $(K, X)$ and $(J, Y)$ are called exotically concordant (resp. topologically concordant) if there is a smooth (resp. topological) $\mathbb{Z}$-homology cobordism $W$ from $X$ to $Y$ with $\pi_{1}(W)$ normally generated by the images of each of $\pi_{1}(X)$ and $\pi_{1}(Y)$ and in which $K$ and $J$ cobound a smooth (resp. locally flat) annulus. The set of all knots in $\mathbb{Z}$-homology spheres modulo exotic concordance (resp. topological concordance) is denoted by $\widehat{\mathcal{C}}_{\text {ex }}$ (resp. $\widehat{\mathcal{C}}_{\text {top }}$ ).

Now, suppose that $R$ is a localization of $\mathbb{Z}$, and $K$ and $J$ are knots in the $R$-homology spheres $X$ and $Y$. Then $(K, X)$ and $(J, Y)$ are called $R$-concordant if there is a smooth $R$-homology cobordism $W$ from $X$ to $Y$ in which $K$ and $J$ cobound a smooth annulus. We denote by $\widehat{\mathcal{C}}_{R}$ the set of all knots in $R$-homology spheres modulo $R$-concordance.

Proposition 2.11 The map

$$
\Psi: \mathcal{C}_{*} \rightarrow \widehat{\mathcal{C}}_{*}, \quad[K] \mapsto\left[\left(K, S^{3}\right)\right]
$$

is well-defined and injective, for each value of $* \in\{$ ex, top, $R\}$.

Proof Let $*=$ ex. If $K$ is exotically concordant to $J$, then $K$ and $J$ cobound a smooth annulus in $W:=S^{3} \times[0,1]$ with a possibly exotic smooth structure. Therefore, $W$ is a homology cobordism from $S^{3}$ to itself, and since $\pi_{1}(W)=0$, we have $\left(K, S^{3}\right)=\left(J, S^{3}\right)$ in $\hat{\mathcal{C}}_{\text {ex }}$. Hence, the map $\Psi$ is well-defined.

Suppose $\left(K, S^{3}\right)=\left(J, S^{3}\right)$ in $\widehat{\mathcal{C}}_{\text {ex }}$. Then $K$ and $J$ cobound a smooth annulus in a smooth homology cobordism $W$ from $S^{3}$ to itself with $\pi_{1}(W)$ normally generated by $\pi_{1}\left(S^{3}\right)=0$. Thus, $W$ is simply connected, and by Freedman's proof of the topological 4-dimensional Poincaré conjecture [9], $W$ is homeomorphic to $S^{3} \times[0,1]$ (but not necessarily diffeomorphic). Thus, $K$ is exotically concordant to $J$.

The proofs in the cases $*=$ top and $*=R$ are similar. 
In fact, $\widehat{\mathcal{C}}_{*}$ has a group structure coming from the connected-sum operation, and the map $\mathcal{C}_{*} \hookrightarrow \widehat{\mathcal{C}}_{*}$ is a monomorphism. Since we are not so interested with the group structure on knot concordance in this paper, we will not prove this fact.

Remark 2.12 We noted in Remark 2.1 that patterns $P_{i} \subseteq V_{i}=S^{1} \times D^{2}(i=0,1)$ in $\mathcal{S}_{\text {str }}$ can be uniquely represented by the 2 -component links $\left(\widetilde{P}_{i}, \eta_{i}\right)$. In fact, if the links $\left(\widetilde{P}_{0}, \eta_{0}\right)$ and $\left(\widetilde{P}_{1}, \eta_{1}\right)$ are exotically (resp. topologically) concordant, then the strong homology cylinders $E\left(P_{0}\right)$ and $E\left(P_{1}\right)$ are strongly (resp. strongly topologically) cobordant. This is seen by cutting out a regular neighborhood of the concordance between the two links in $S^{3} \times[0,1]$. Similarly, suppose the patterns $P_{i}$ are in $\mathcal{S}_{R}$ for some localization $R$ of $\mathbb{Z}$; if the links $\left(\widetilde{P}_{0}, \eta_{0}\right)$ and $\left(\tilde{P}_{1}, \eta_{1}\right)$ are $R$-concordant, then $E\left(P_{0}\right)$ and $E\left(P_{1}\right)$ are $R$-cobordant.

Remark 2.13 A generalized $*$-pattern $\left(M, i_{+}, i_{-}\right)$yields a link $\left(\widetilde{P}_{M}, \eta_{M}\right)$ in a $3-$ manifold $\hat{M}$, as follows. We obtain $\widehat{M}$ by attaching $2-$ handles to $M$ along $i_{-}(m)$ and $i_{+}(\ell)$, followed by $3-$ handles along the two resulting sphere boundary components. Let $\widetilde{P}_{M}$ denote the image of $i_{-}(\ell)$ in $\hat{M}$ and $\eta_{M}$ the image of $i_{+}(m)$ in $\hat{M}$. If $*=R$, then $\hat{M}$ is an $R$-homology sphere, whereas if $*=$ str, then $\hat{M}$ is a $\mathbb{Z}$-homology sphere. It is straightforward to see that if two $R$-cylinders $\left(M, i_{+}, i_{-}\right)$and $\left(N, j_{+}, j_{-}\right)$ are $R$-cobordant, then $\left(\widetilde{P}_{M}, \eta_{M}\right)$ and $\left(\widetilde{P}_{N}, \eta_{N}\right)$ are therefore concordant as links in an $R$-homology cobordism from $\hat{M}$ to $\hat{N}$. If $M$ and $N$ are strong homology cylinders which are strongly cobordant, then the links $\left(\widetilde{P}_{M}, \eta_{M}\right)$ and $\left(\widetilde{P}_{N}, \eta_{N}\right)$ are concordant in a homology cobordism from $\hat{M}$ to $\widehat{N}$ whose fundamental group is normally generated by each of $\pi_{1}(\hat{M})$ and $\pi_{1}(\hat{N})$. If the strong homology cylinders are merely strongly topologically cobordant, then the link concordance is topological.

\subsection{Generalized patterns act on knots in homology spheres via general- ized satellite operators}

Proposition 2.14 The monoid $\widehat{\mathcal{S}}_{R}^{0}$ acts on the set of knots in $R$-homology spheres. For the map $E: \mathcal{S}_{R} \rightarrow \hat{\mathcal{S}}_{R}^{0}$, if $P \in \mathcal{S}_{R}$ and $K \subseteq S^{3}$ is a knot, $\left(P(K), S^{3}\right)$ is isotopic to $(E(P))\left(K, S^{3}\right)$.

Note that since $\hat{\mathcal{S}}_{\text {str }}^{0} \subseteq \widehat{\mathcal{S}}_{\mathbb{Z}}^{0}$, the above says that $\hat{\mathcal{S}}_{\text {str }}^{0}$ acts on the set of knots in $\mathbb{Z}-$ homology spheres.

Proof of Proposition 2.14 Let $K$ be a knot in the $R$-homology sphere $Y$, and $\left(M, i_{+}, i_{-}\right)$be an $R$-homology cylinder. Construct a 3 -manifold $Y^{\prime}$ as follows - 
start with $M$, glue on a solid torus along $i_{-}(T)$ such that $i_{-}(m)$ bounds a disk, and glue in $Y-N(K)$ such that $i_{+}(\ell) \sim \ell(K)$ and $i_{+}(m) \sim m(K)$. Therefore,

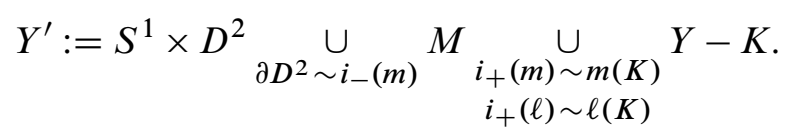

It is easy to check that $Y^{\prime}$ is an $R$-homology sphere when $\left(V, i_{+}, i_{-}\right) \in \widehat{\mathcal{S}}_{R}^{0}$. Let $K^{\prime}$ be the core of the solid torus $S^{1} \times D^{2}$ in this decomposition. The above construction gives the desired action on knots in $R$-homology spheres; that is,

$$
\left(M, i_{+}, i_{-}\right) \cdot(K, Y):=\left(K^{\prime}, Y^{\prime}\right) .
$$

It is straightforward to see that for all homology cylinders $M$ and $N$ and any pair $(K, Y)$ as above, $(M \star N) \cdot(K, Y)=M \cdot(N \cdot(K, Y))$. Therefore, we have a monoid action. The pairs $\left(P(K), S^{3}\right)$ and $(E(P)) \cdot\left(K, S^{3}\right)$ are isotopic since the gluing instructions given above are identical to those in the classical satellite construction.

Proposition 2.15 Let $R$ be a localization of $\mathbb{Z}$ and $* \in\{$ ex, top, $R\}$. The monoid action of Proposition 2.14 descends to a group action by $\widehat{\mathcal{S}}_{*}$ on $\widehat{\mathcal{C}}_{*}$ for each choice of $* \in\{\mathrm{ex}, \mathrm{top}, R\}$.

Proof Let $\left(M, i_{+}, i_{-}\right),\left(N, j_{+}, j_{-}\right) \in \mathcal{S}_{*}$ be generalized $*$-patterns, for $* \in\{\operatorname{str}, R\}$, and $K$ and $J$ be knots in the manifolds $Y$ and $X$, respectively. From the proof of Proposition 2.14, we know that $\left(M, i_{+}, i_{-}\right) \cdot(K, Y)$ and $\left(N, j_{+}, j_{-}\right) \cdot(J, X)$ are knots in the 3-manifolds $Y^{\prime}=S^{1} \times D^{2} \cup M \cup S^{3}-K$ and $X^{\prime}=S^{1} \times D^{2} \cup N \cup S^{3}-J$, where, in particular, the resulting knots are given by the cores of the $S^{1} \times D^{2}$-pieces.

Suppose $(K, Y)$ and $(J, X)$ are $R$-concordant, ie $(K, Y)=(J, X)$ in $\widehat{\mathcal{C}}_{R}$, and $\left(M, i_{+}, i_{-}\right)$and $\left(N, j_{+}, j_{-}\right)$are $R$-cobordant, ie $\left(M, i_{+}, i_{-}\right)=\left(N, j_{+}, j_{-}\right)$in $\widehat{\mathcal{S}}_{R}$. Then there is an $R$-cobordism $U_{0}$ between $M$ and $N$, and an $R$-concordance $C$ from $K$ to $J$ in some 4-manifold; let $E(C)$ be the complement of $C$.

The gluing instructions used to build $X^{\prime}$ and $Y^{\prime}$ extend to gluing instructions for a 4-manifold

$$
U=S^{1} \times D^{2} \times[0,1] \cup U_{0} \cup E(C)
$$

with $\partial U=Y^{\prime} \sqcup-X^{\prime}$. Since each of the pieces of $U$ is smooth, $U$ is smooth as well. Moreover, since each of the pieces of $U$ is an $R$-homology cobordism, it follows from a Mayer-Vietoris argument that $U$ is an $R$-homology cobordism as well, where the core of $S^{1} \times D^{2} \times[0,1]$ is a smooth annulus cobounded by the two resulting knots. This completes the proof in the case that $*=R$. 
For the $*=$ ex case, we only need to show that the additional condition on fundamental groups is satisfied when the spaces used above are in $\widehat{\mathcal{S}}_{\text {ex }}$ and $\widehat{\mathcal{C}}_{\text {ex }}$. This can be seen using two successive Seifert-van Kampen arguments, since the fundamental group of each piece of $U$ is normally generated by each of its boundary components. The last remaining case, $*=$ top, follows from the various arguments above, with the additional trivial observation that if the pieces of $U$ are merely topological, the core of $S^{1} \times D^{2} \times[0,1]$ is a locally flat annulus.

Thus, any generalized $*$-pattern $\left(M, i_{+}, i_{-}\right)$yields an induced function

$$
M: \widehat{\mathcal{C}}_{*} \rightarrow \widehat{\mathcal{C}}_{*}
$$

as described above, which we will call a generalized satellite operator, for appropriate choice of symbols (generalized str-patterns induce functions on $\widehat{\mathcal{C}}_{\text {ex }}$ and $\widehat{\mathcal{C}}_{\text {top }}$ while generalized $R$-patterns induce functions on $\hat{\mathcal{C}}_{R}$, where $R$ is a localization of $\mathbb{Z}$ ).

We combine the results and definitions of this section to give the main theorem.

Main theorem Let $R$ be a localization of $\mathbb{Z}$. For the maps $\Psi$ from Proposition 2.11, the monoid morphisms $E: \mathcal{S}_{\text {str }} \rightarrow \widehat{\mathcal{S}}_{\text {ex }}, \mathcal{S}_{\text {str }} \rightarrow \widehat{\mathcal{S}}_{\text {top }}$ and $\mathcal{S}_{R} \rightarrow \widehat{\mathcal{S}}_{R}$ from Proposition 2.9, and any $P \in \mathcal{S}_{\text {str }}$, and $Q \in \mathcal{S}_{R}$, the following diagrams commute.
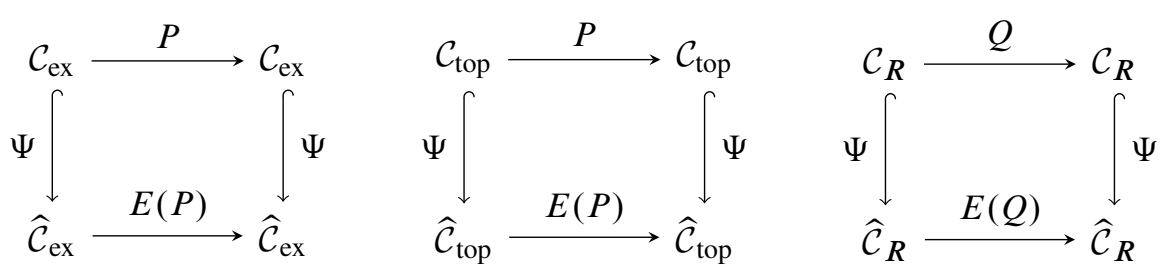

Proof The result follows from Propositions 2.9, 2.11, and 2.14.

As an immediate corollary of the main theorem we recover the following result from [5].

Corollary 2.16 [5, Theorem 5.1] Let $P$ be a pattern. If $P$ has winding number $n \neq 0$, then $P: \mathcal{C}_{\mathbb{Z}[1 / n]} \rightarrow \mathcal{C}_{\mathbb{Z}[1 / n]}$ is injective. If $P$ has strong winding number \pm 1, then $P: \mathcal{C}_{\text {ex }} \rightarrow \mathcal{C}_{\text {ex }}$ and $P: \mathcal{C}_{\text {top }} \rightarrow \mathcal{C}_{\text {top }}$ are injective.

Proof The proof is a straightforward diagram chase. Let $* \in\left\{\right.$ ex, top, $\left.\mathbb{Z}\left[\frac{1}{n}\right]\right\}$. Suppose that $P(K)$ is concordant to $P(J)$ in the $*$-category; ie $P(K)=P(J)$ in $\mathcal{C}_{*}$. Then $\Psi(P(K))=\Psi(P(J))$. Since the diagrams in (2-1) commute, we see that $(E(P))(\Psi(K))=(E(P))(\Psi(J))$. Since $E(P) \in \hat{\mathcal{S}}_{*}$ is an element of a group which acts on $\widehat{\mathcal{C}}_{*}$, it has an inverse. Therefore, the satellite operator $E(P)$ is bijective and, in particular, injective. Thus, $\Psi(K)=\Psi(J)$. But $\Psi$ is also injective; therefore, we conclude that $K=J$ in $\mathcal{C}_{*}$, as needed. 


\section{Surjectivity of satellite operators}

Since satellite operators have now been shown to be injective in several categories (in Section 2 as well as in [5]), it is natural to ask which satellite operators $P: \mathcal{C}_{*} \rightarrow$ $\mathcal{C}_{*}$ are surjective, for $* \in\{\mathrm{ex}$, top, $R\}$ and a localization $R$ of $\mathbb{Z}$. The following proposition shows that only patterns of winding number \pm 1 may induce surjective satellite operators.

Proposition 3.1 Let $P$ be a pattern with winding number $n \neq \pm 1$. The induced satellite operator $P: \mathcal{C}_{*} \rightarrow \mathcal{C}_{*}$ is not surjective for any $* \in\{$ ex, top, $R\}$, where $R$ is a localization of $\mathbb{Z}$.

Proof We know from $[16 ; 17]$ that for any knot $K$,

$$
\sigma(P(K), \omega)=\sigma(P(U), \omega)+\sigma\left(K, \omega^{n}\right),
$$

where $U$ is the unknot, and $\sigma(\cdot, \omega)$ denotes the Levine-Tristram signature at $\omega \in \mathbb{C}$, with $|\omega|=1$. For a fixed $P$, this imposes restrictions on the signature function of $P(K)$, as follows. Let $J$ be a knot for which $\sigma(J, \omega)$ does not have the form $g\left(\omega^{n}\right)$ for any function on $g$ on $S^{1}$ (for example, the right-handed trefoil knot). Then $\sigma(P(U) \# J, \omega)=\sigma(P(U), \omega)+\sigma(J, \omega)$ cannot be of the form prescribed to $\sigma(P(K), \omega)$ in Equation (3-1). Therefore, $P(U) \# J$ is not in the image of $P$; the result follows, since the signature function is an invariant of rational concordance.

As a result, we mostly restrict ourselves, henceforth, to patterns in $\mathcal{S}_{\mathbb{Z}}$ and $\mathcal{S}_{\text {str }}$. Of course, connected-sum patterns, ie patterns of the form $Q_{J}$ shown in Figure 5, are clearly surjective. We say that a pattern $P$ with winding number \pm 1 is nontrivial if it is distinct as an element of $\hat{\mathcal{S}}_{\mathbb{Z}}$ from the connected-sum patterns $Q_{J}$, for all knots $J$. We first note that we have a characterization of patterns inducing surjective satellite operators as follows.

Proposition 3.2 The pattern $P \in \mathcal{S}_{\text {str }}$ induces a surjective map $P: \mathcal{C}_{*} \rightarrow \mathcal{C}_{*}$ for $* \in\{$ ex, top $\}$ if and only if $E(P)^{-1}\left(\Psi\left(\mathcal{C}_{*}\right)\right) \subseteq \Psi\left(\mathcal{C}_{*}\right)$, where $E(P)^{-1}$ is the inverse of the homology cylinder $E(P) \in \widehat{\mathcal{S}}_{*}$. Similarly, $P \in \mathcal{S}_{\mathbb{Z}}$ induces a surjective map $P: \mathcal{C}_{\mathbb{Z}} \rightarrow \mathcal{C}_{\mathbb{Z}}$ if and only if $E(P)^{-1}\left(\Psi\left(\mathcal{C}_{\mathbb{Z}}\right)\right) \subseteq \Psi\left(\mathcal{C}_{\mathbb{Z}}\right)$.

Proof The key observation here is that since $E(P)$ acts via a group action, it must induce a bijection on $\widehat{\mathcal{C}}_{*}$ for each $* \in\{$ ex, top, $\mathbb{Z}\}$. Therefore, by the commutativity of the diagrams in (2-1) and the injectivity of $\Psi$, we see that $P: \mathcal{C}_{*} \rightarrow \mathcal{C}_{*}$ is surjective if and only if $E(P)^{-1}\left(\Psi\left(\mathcal{C}_{*}\right)\right)=\Psi\left(\mathcal{C}_{*}\right)$. However, we know that $E(P)\left(\Psi\left(\mathcal{C}_{*}\right)\right) \subseteq \Psi\left(\mathcal{C}_{*}\right)$ for all $P$. 
It is worth noting that one way to guarantee that $E(P)^{-1}\left(\Psi\left(\mathcal{C}_{*}\right)\right) \subseteq \Psi\left(\mathcal{C}_{*}\right)$, for $* \in$ $\{$ ex, top $\}$, is for $E(P)^{-1}$ to be the image under $E: \mathcal{S}_{\text {str }} \rightarrow \widehat{\mathcal{S}}_{*}$ of some $\bar{P} \in \mathcal{S}_{\text {str }}$, since as we saw in the proof above, $E(\bar{P})\left(\Psi\left(\mathcal{C}_{*}\right)\right) \subseteq \Psi\left(\mathcal{C}_{*}\right)$ for all $\bar{P}$. This holds for connected-sum patterns as shown below.

Proposition 3.3 For $* \in\{$ top, ex, $R\}$ and any knot $J$ in $S^{3}$, we have $E\left(Q_{J}\right)^{-1}=$ $E\left(Q_{-J}\right)$ in $\widehat{\mathcal{S}}_{*}$.

Proof To prove the result is suffices to find a strong cobordism from $E\left(Q_{J}\right) \star$ $E\left(Q_{-J}\right)$ to the identity element $(T \times[0,1], \mathrm{id} \times\{0\}$, id $\times\{1\})$. Since $E: \mathcal{S}_{*} \rightarrow \widehat{\mathcal{S}}_{*}$ is a homomorphism $E\left(Q_{J}\right) \star E\left(Q_{-J}\right)=E\left(Q_{J} \star Q_{-J}\right)$. Finally, it is easy to see from the definition of multiplication in $\mathcal{S}_{*}$ that $Q_{J} \star Q_{-J}=Q_{J \#-J}$.

As a 3-manifold $E\left(Q_{J \#-J}\right)$ is diffeomorphic to the complement in $S^{3}$ of the 2component link $L$ consisting of $J \#-J$ and a meridian $\mu$ for $J \#-J$. The diffeomorphism sends the longitude and meridian of $J \#-J$ to the longitude and meridian of $Q_{J \#-J}$ respectively, and the meridian and longitude of $\mu$ to the longitude and meridian of the solid torus $\ell(V)$ and $m(V)$ respectively.

Finally, since $J \#-J$ is slice, the link $(J \#-J) \sqcup \mu$ is concordant to the Hopf link, whose exterior is diffeomorphic to $T \times[0,1]$. It is straightforward to check that the complement of the concordance provides a $*$-cobordism between $E\left(Q_{J} \star Q_{-J}\right)$ and the identity element $(T \times[0,1], \mathrm{id} \times\{0\}, \mathrm{id} \times\{1\})$.

In fact, there exist nontrivial patterns $P$ with winding number \pm 1 such that $E(P)^{-1}=$ $E(\bar{P})$ for some $\bar{P} \in \mathcal{S}_{\mathbb{Z}}$, as we see below.

Theorem 3.4 Let $P \subseteq V=S^{1} \times D^{2}$ be in $\mathcal{S}_{\mathbb{Z}}$. If $m(P)$ is in the normal subgroup of $\pi_{1}(E(P))$ generated by $m(V)$ then $P$ has strong winding number \pm 1 and there exists another strong winding number one pattern $\bar{P}$ such that $E(P)^{-1}=E(\bar{P})$ as homology cylinders.

Proof We see that $P$ has strong winding number \pm 1 by Proposition 2.4. Indeed, in order to construct $S^{3}-\widetilde{P}$ from $P$, a 2 -handle is added to $\ell(V)$. Thus, $\pi_{1}(E(P)) \rightarrow$ $\pi_{1}\left(S^{3}-\widetilde{P}\right)$ is surjective. By assumption, $m(P)$ is in the normal subgroup generated by $m(V)$ in $\pi_{1}(E(P))$. Since $\pi_{1}(E(P)) \rightarrow \pi_{1}\left(S^{3}-\widetilde{P}\right)$ is surjective, $m(P)$ is in the normal subgroup generated by $m(V)=\eta$ in $\pi_{1}\left(S^{3}-\widetilde{P}\right)$. Since $\widetilde{P}$ is a knot in $S^{3}$, we know $\pi_{1}\left(S^{3}-\widetilde{P}\right)$ is normally generated by $m(P)$. Since $m(P) \in\langle\langle\eta\rangle\rangle$, it follows that $\eta$ normally generates $\pi_{1}\left(S^{3}-\tilde{P}\right)$. Proposition 2.4 now concludes that $P$ has strong winding number \pm 1 . 
Note that $\pi_{1}(E(P)) /\langle\langle m(P)\rangle\rangle \cong \pi_{1}(V) \cong \mathbb{Z}$ since the solid torus $V$ is obtained from $E(P)$ by adding a 2-handle to the meridian of $P$ and then a 3-handle. Additionally, $m(V)$ is nullhomotopic in $V$ so that $m(V)=0$ in $\pi_{1}(E(P)) /\langle\langle m(P)\rangle\rangle$ and $\langle\langle m(V)\rangle\rangle \subseteq$ $\langle\langle m(P)\rangle$. By assumption, $m(P) \in\langle\langle m(V)\rangle\rangle$ so that we conclude that $\langle\langle m(P)\rangle\rangle=$ $\left\langle\langle m(V)\rangle\right.$. Therefore, $\pi_{1}(E(P)) /\langle\langle m(P)\rangle\rangle=\pi_{1}(E(P)) /\langle\langle m(V)\rangle\rangle \cong \mathbb{Z}$.

Now consider $E(P)^{-1}$. By definition, $E(P)^{-1}=\left(-E(P), i_{-}, i_{+}\right)$. Perform a Dehn filling on $-E(P)$ along $m(V)$ to obtain a manifold $X$. By the preceding paragraph, $\pi_{1}(X) \cong \mathbb{Z}$ and therefore, since $\partial X$ has no $S^{2}$ components, $X$ is diffeomorphic to the solid torus [12, Theorem 5.2]. Since $m(P)$ must be mapped to a curve which is null homologous, we see that $m(P) \mapsto 1 \times \partial D^{2}$. By performing meridional twists if necessary, we may assume that $\ell(P) \mapsto S^{1} \times 1$. Then, by definition, if we denote by $\bar{P}$ the image of $\ell(V)$ in $X \cong S^{1} \times D^{2}$, we see that $E(\bar{P})=\left(-E(P), i_{-}, i_{+}\right)$. Since $\ell(V)$ is homologous to $\ell(P)$ in $E(P)$ (since $P \in \mathcal{S}_{\mathbb{Z}}$ ), $w(\bar{P})=w(P)=1$. Since $P$ has strong winding number \pm 1 each of the sets $\{m(P), \ell(P)\}$ and $\{m(V(P)), \ell(V(P))\}$ normally generate $\pi_{1}(E(P))$. But these sets of curves are respectively the same as $\{m(V(\bar{P})), \ell(V(\bar{P}))\}$ and $\{m(\bar{P}), \ell(\bar{P})\}$. It follows that $\bar{P}$ has strong winding number \pm 1 as well.

Under the assumptions of the above theorem, the pattern $P$ has an inverse $\bar{P}$ which is also a pattern. A close reading of the proof of the theorem reveals how to draw a picture of the latter given the former. In fact, it is easier to see how to draw a picture of the corresponding 2-component link $\bar{L}$ (see Remark 2.1 and Figure 3); recall that given such a link $\bar{L}$ we can recover the pattern $\bar{P}$ by removing a tubular neighborhood of the second component of $\bar{L}$ from $S^{3}$.

Start with the 2-component link $L$ corresponding to the given pattern $P$. The manifold $E(P)$ is exactly the complement of this link in $S^{3}$. A key observation in the above proof is that the manifold obtained by performing a Dehn filling of $-\left(S^{3}-L\right)$ along the second component of $L$ is homeomorphic to a solid torus, via a homeomorphism taking the first component of $L$ to the longitude of the solid torus. Of course, if we were to perform a Dehn filling along the longitude of a solid torus we obtain $S^{3}$. Therefore, this is the same as saying that if we reverse the orientation and crossings of $L$ and then perform 0 -framed Dehn surgery on $S^{3}$ along both components, we get back $S^{3}$. Further, in this new $S^{3}$, we can find the components of the link corresponding to $\bar{P}$. In the proof above these were the images of the curves $\ell(V)$ and $m(P)$. In the framework of links, these are the images of the meridians of the two components of $L$ (see Figure 6) - the meridian of the first component of $L$ is the second component of $\bar{L}$ and the meridian of the second component of $L$ is the first component of $\bar{L}$. Therefore, we have proved the following proposition. 


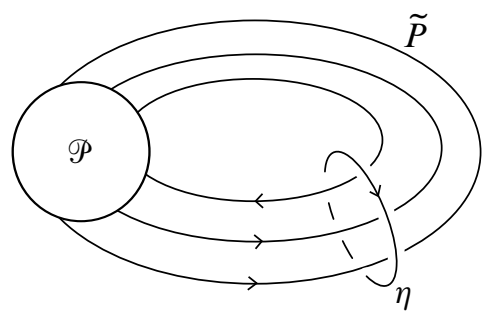

(a)

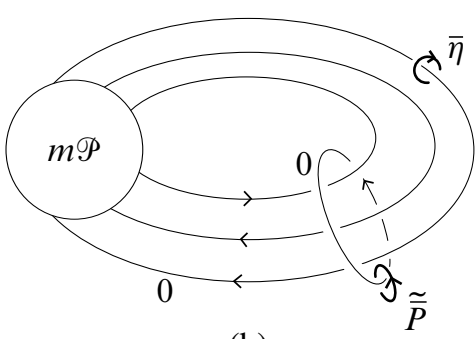

(b)

Figure 6: (a) The 2-component link $(\widetilde{P}, \eta)$ corresponds to the pattern $P$; see Figure 3 . Recall that the circle containing $\mathscr{P}$ denotes a tangle. (b) The circle containing $m \mathscr{P}$ indicates the tangle obtained from $\mathscr{P}$ in the previous panel by reversing all the crossings. The curves decorated with zeros give a surgery diagram for $S^{3}$. The 2-component link $(\widetilde{\bar{P}}, \bar{\eta})$ (drawn in heavier weight) corresponds to the pattern $\bar{P}$.

Proposition 3.5 Let $P \subseteq V=S^{1} \times D^{2}$ be in $\mathcal{S}_{\mathbb{Z}}$. Assume that $m(P)$ is in the normal subgroup of $\pi_{1}(E(P))$ generated by $m(V)$ and $(\widetilde{P}, \eta)$ is the 2-component link corresponding to $P$ where $\eta$ is unknotted in $S^{3}$. Then the inverse of $P$ is given by the link $(\widetilde{\bar{P}}, \bar{\eta})$ in the surgery diagram for $S^{3}$ given by zero surgery on both components of the reverse mirror image of $(\widetilde{P}, \eta)$, where $\widetilde{\bar{P}}$ is the meridian of $\eta$ and $\bar{\eta}$ is the meridian of $\widetilde{P}$.

Remark 3.6 Let $P_{m} \subseteq V\left(P_{m}\right)=S^{1} \times D^{2}$ be the patterns shown in Figure 2 and again in Figure 7 (these first appeared in [1]). Note that each $P_{m}$ satisfies the requirements of Theorem 3.4, as follows. It suffices to show that $m\left(P_{m}\right)$ is nullhomotopic in the 3-manifold $N$ obtained from $E\left(P_{m}\right)$ by adding a 2-handle along $m\left(V\left(P_{m}\right)\right)$. The result of sliding $P_{m}$ over this 2-handle twice (isotopies in $N$ ) is depicted in Figure 7. In the result of the isotopy, the meridian of $P_{m}$ cobounds an annulus with the meridian of $V\left(P_{m}\right)$ and so bounds a disk in $N$. As a result, we can use Proposition 3.5 to construct inverses for the patterns $\left\{P_{m}\right\}_{m \geq 0}$ shown in Figure 2. This is indicated in Figure 8.

Theorem 3.4 also gives a sufficient condition for patterns to induce bijective satellite operators as follows.

Corollary 3.7 Let $P \subseteq V=S^{1} \times D^{2}$ be in $\mathcal{S}_{\mathbb{Z}}$. If $m(P)$ is in the normal subgroup of $\pi_{1}(E(P))$ generated by $m(V)$ then $P: \mathcal{C}_{*} \rightarrow \mathcal{C}_{*}$ is bijective for $* \in\{$ ex, top, $\mathbb{Z}\}$.

Proof Surjectivity follows from Proposition 3.2 and Theorem 3.4 since if $E(P)^{-1}=$ $E(\bar{P})$ for some $\bar{P} \in \mathcal{S}_{\mathbb{Z}}$, then $E(P)^{-1}\left(\Psi\left(\mathcal{C}_{*}\right)\right)=(E(\bar{P}))\left(\Psi\left(\mathcal{C}_{*}\right)\right) \subseteq \Psi\left(\mathcal{C}_{*}\right)$. Any 
$P \in \mathcal{S}_{\mathbb{Z}}$ is injective on $\mathcal{C}_{\mathbb{Z}}$. By Theorem $3.4, P$ has strong winding number \pm 1 and therefore, induces injective satellite operators on $\mathcal{C}_{\text {ex }}$ and $\mathcal{C}_{\text {top }}$.

Before we provide the promised examples of bijective satellite operators, we will need the following lemma, which provides an extension of the operation $P \mapsto \tau(P)$ of "twisting a pattern" to the setting of generalized patterns. The function $\tau: \mathcal{S} \rightarrow \mathcal{S}$ gives a full right-handed twist to each pattern, as shown in Figure 9.

Lemma 3.8 For $* \in\{$ ex, top, $\mathbb{Z}\}$ there is a map $\hat{\tau}: \widehat{\mathcal{S}}_{*} \rightarrow \widehat{\mathcal{S}}_{*}$ such that for all $P \in \mathcal{S}_{\text {str }}$, $\hat{\tau}(E(P))=E(\tau(P))$ as elements of $\widehat{\mathcal{S}}_{*}$.

Proof Let $P$ be a pattern with winding number \pm 1 in the solid torus $V$, and $\tau(P)$ be the corresponding twisted pattern. Let $f: V \rightarrow V$ be the homeomorphism given by a negative meridional Dehn twist. Notice that $f$ sends $\tau(P)$ to $P$. Thus, $f$ restricts to a homeomorphism $E(\tau(P)) \rightarrow E(P)$. This homeomorphism sends $m(V)$ to $m(V)$ and $\ell(V)$ to $\ell(V)-m(V)$. Since $f_{*}$ is well-defined on homology classes, $f$ sends $m(\tau(P))$ to $m(P)$ and $\ell(\tau(P))$ to $\ell(P)-m(P)$. Let $\phi: T \rightarrow T$ be the homeomorphism of the torus sending $m$ to $m$ and $\ell$ to $\ell-m$. As homology cylinders, $P$ and $\tau(P)$ are given by $\left(E(P), i_{+}, i_{-}\right)$and $\left(E(P), j_{+}, j_{-}\right)$where $j_{\epsilon}=i_{\epsilon} \circ \phi$ for $\epsilon \in\{+,-\}$.

For any homology cylinder $\left(M, i_{+}, i_{-}\right)$, define $\hat{\tau}\left(M, i_{+}, i_{-}\right)=\left(M, i_{+} \circ \phi, i_{-} \circ \phi\right)$. By the preceding paragraph, for any pattern $P, \hat{\tau}(E(P))=E(\tau(P))$. It remains only to show that $\hat{\tau}$ is well-defined modulo $*$-cobordism. Assume that $W$ is a $*$-cobordism between $\left(M, i_{+}, i_{-}\right)$and $\left(N, j_{+}, j_{-}\right)$. Taking advantage of the fact that $\phi: T \rightarrow T$ is a diffeomorphism, we see that

$$
\begin{aligned}
\partial W & =M \sqcup-N / i_{+}(x)=j_{+}(x), i_{-}(x)=j_{-}(x), \forall x \in T \\
& \cong M \sqcup-N / i_{+}(\phi(x))=j_{+}(\phi(x)), i_{-}(\phi(x))=j_{-}(\phi(x)), \forall x \in T .
\end{aligned}
$$

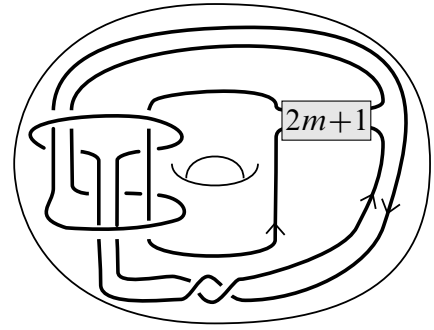

The satellite operator $P_{m}$.

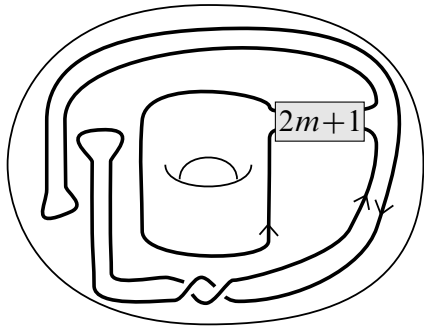

The result of sliding $P_{m}$ over the meridian of $V(m)$.

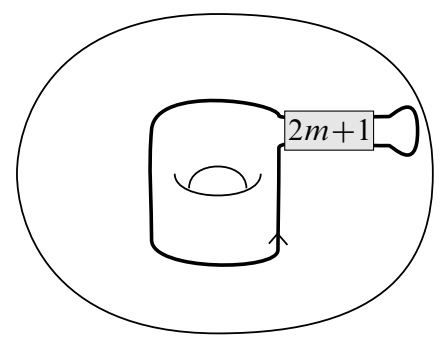

The result of a further isotopy.

Figure 7: The patterns $P_{m}$ satisfy the requirements of Theorem 3.4. 

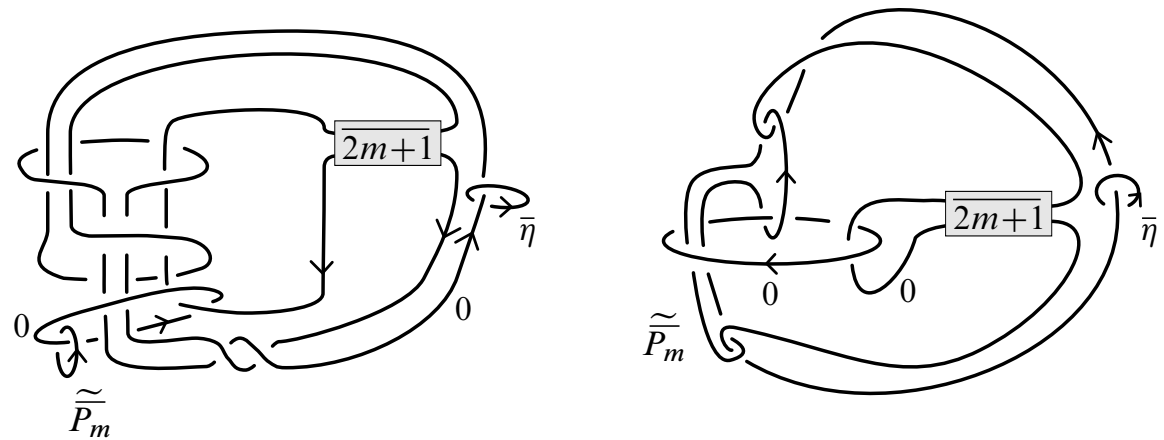

$$
\sqrt{2 m+1}=\frac{2 m+1 \text { half-twists }}{2 m}
$$

Figure 8: Left: By Proposition 3.5, the link $\left(\widetilde{\overline{P_{m}}}, \bar{\eta}\right)$ in this surgery diagram represents the inverse of the pattern $P_{m}$, for $m \geq 0$. Right: This diagram is obtained from the one in the previous panel by handle-slides and isotopy, and we see a standard picture of $S^{3}$ (notice that the curves marked with zeros form a Hopf link). To get a picture of the inverse pattern as a link, we simply need to slide the undecorated curves away from the surgery curves. This readily yields a picture of a link in $S^{3}$.

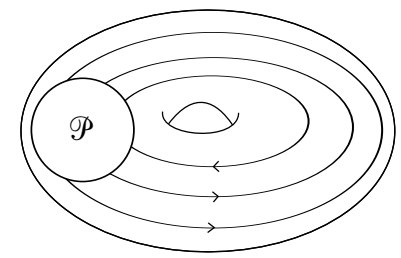

A pattern $P$.

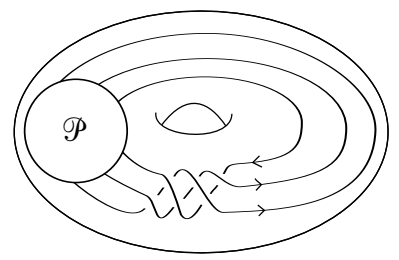

The twisted pattern $\tau(P)$.

Figure 9: Twisting a pattern.

Therefore, $W$ is also a $*-$ cobordism between $\hat{\tau}\left(M, i_{+}, i_{-}\right)$and $\hat{\tau}\left(N, j_{+}, j_{-}\right)$.

We are now ready to construct examples of patterns which yield bijective satellite operators on knot concordance, and are distinct from connected-sum patterns.

Corollary 3.9 Fix $m \geq 0$. The pattern $P_{m} \subseteq V\left(P_{m}\right)=S^{1} \times D^{2}$ shown in Figure 2 (and again in Figure 7) induces a bijective satellite operator $P_{m}: \mathcal{C}_{*} \rightarrow \mathcal{C}_{*}$ for $* \in$ $\{$ ex, top, $\mathbb{Z}\} ;$ moreover, as elements of $\widehat{\mathcal{S}}_{\mathbb{Z}}$, we have $E\left(P_{m}\right) \neq E\left(Q_{J}\right)$ for all knots $J$.

Proof We already know that each $P_{m}$ satisfies the requirements of Theorem 3.4 and Corollary 3.7 from Remark 3.6. This gives the first statement. 
In order to see the second result notice that the twisting map $\tau$ of Figure 9 and Lemma 3.8 sends $Q_{J}$ to $Q_{J}$ for any knot $J$. It suffices then to prove that $\tau\left(P_{m}\right) \neq P_{m}$ in $\hat{\mathcal{S}}_{\mathbb{Z}}$. In order to see this first observe that $P_{m}(U)$ is smoothly slice. However, according to an Alexander polynomial computation the knots $\left(\tau\left(P_{m}\right)\right)(U)$ are not slice for $m \geq 0$; see also [1, Theorem 3.6]. Since the Alexander polynomial is an obstruction to being slice in a $\mathbb{Z}$-homology sphere, we conclude that as maps on $\mathcal{C}_{\mathbb{Z}}, P_{m}$ and $\tau\left(P_{m}\right)$ disagree, so that as elements of $\widehat{\mathcal{S}}_{\mathbb{Z}}$, we have $P_{m} \neq \tau\left(P_{m}\right)$. This completes the proof.

In passing, we note that by Remark 2.12, the above result implies that the link $\left(P_{m}, \eta\left(V\left(P_{m}\right)\right)\right)$ is not (smoothly, exotically, topologically, or $\mathbb{Z}-$ ) concordant to the link $\left(Q_{J}, m\left(V\left(Q_{J}\right)\right)\right)$ for any knot $J$ and $m \geq 0$ (see also [5, Proposition 2.3]). It is also worth noting that even though $P_{m}$ and $Q_{J}$ are distinct as homology cylinders for all knots $J$ and $m \geq 0$, it is still possible that they induce the same satellite operator, ie $P_{m}(K)=J \# K$ for some fixed knot $J$, and any knot $K$.

We end this section with the following result, leading to a corollary for satellite operators induced by patterns with winding number other than \pm 1 .

Proposition 3.10 There exists a pattern in $\mathcal{S}_{\text {str }}$ for which the induced satellite operator on $\mathcal{C}_{*}$ is not surjective if and only if there exists a pattern in $\mathcal{S}_{\text {str }}$ for which the unknot is not in the image of the induced satellite operator on $\mathcal{C}_{*}$, for $* \in\{\mathrm{ex}, \operatorname{top}\}$.

Similarly, for a localization $R$ of $\mathbb{Z}$, there exists a pattern in $\mathcal{S}_{R}$ for which the induced satellite operator on $\mathcal{C}_{R}$ is not surjective if and only if there exists a pattern in $\mathcal{S}_{R}$ for which the unknot is not in the image of the induced satellite operator on $\mathcal{C}_{R}$.

Proof For the forward direction, let $P$ be a pattern that induces a nonsurjective satellite operator on $\mathcal{C}_{*}$ for some $*$, ie there exists a knot $J$ such that $P(K)$ is not concordant to $J$, in the appropriate sense dictated by the value of $*$, for any knot $K$. Then the pattern $Q_{-J} \star P$ does not have the unknot in the image of its induced map on $\mathcal{C}_{*}$, since if $\left(Q_{-J} \star P\right)(K)=-J \# P(K)$ were concordant to the unknot for some $K$, then $P(K)$ would be concordant to $J$. The backward direction is trivial.

Corollary 3.11 For any integer $n$, with $|n|>1$ and $R=\mathbb{Z}\left[\frac{1}{n}\right]$, there exist patterns in $\mathcal{S}_{R}$ for which the induced satellite operator on $\mathcal{C}_{R}$ does not have the unknot in its image.

\section{Concordance to knots in $S^{3}$ and surjectivity of satellite op- erators}

Akbulut conjectured that there exists a winding number one pattern $P$ for which the induced satellite operator on $\mathcal{C}_{\mathrm{ex}}$ does not have the unknot in its image. By 
Proposition 3.10 this conjecture is equivalent to the conjecture that not all satellite operators induced by winding number one patterns are surjective. We restate Akbulut's conjecture in these terms.

Conjecture 4.1 [13, Problem 1.45] There is a pattern with winding number one, $P$, such that the induced satellite operator $P: \mathcal{C}_{*} \rightarrow \mathcal{C}_{*}$ is not surjective, for $* \in\{$ ex, top $\}$.

Consider a knot $K$ in a homology sphere $M$. Then $(K, M)$ gives a class in $\widehat{\mathcal{C}}_{*}$ for $* \in\{$ ex, top $\}$ and one may ask whether there is some knot $K^{\prime} \subseteq S^{3}$ such that $(K, M)$ and $\left(K^{\prime}, S^{3}\right)$ are equivalent in $\widehat{\mathcal{C}}_{*}$. In the PL category, this forms Problem 1.31 of [13]. We restate this as a conjecture.

Conjecture 4.2 [13, Problem 1.31] The image of $\Psi: \mathcal{C}_{*} \rightarrow \widehat{\mathcal{C}}_{*}$ is the set of all concordance classes $(K, M)$ of knots $K$ in 3-manifolds $M$, where $M$ is *-cobordant to $S^{3}$.

We can use the group action given in the main theorem to prove the following relationship between the two conjectures above.

Proposition 4.3 For $P \in \mathcal{S}_{\text {str }}$ and any $K \in \mathcal{C}_{\text {ex }}\left(\operatorname{resp} . \mathcal{C}_{\text {top }}\right)$, if $K \notin \operatorname{Im}\left(P: \mathcal{C}_{\text {ex }} \rightarrow \mathcal{C}_{\text {ex }}\right)$ (resp. $\operatorname{Im}\left(P: \mathcal{C}_{\text {top }} \rightarrow \mathcal{C}_{\text {top }}\right)$ ), then the knot $E(P)^{-1}(\Psi(K))$ is not in the image of $\Psi: \mathcal{C}_{\text {ex }} \rightarrow \widehat{\mathcal{C}}_{\text {ex }}$ (resp. $\mathcal{C}_{\text {top }} \rightarrow \widehat{\mathcal{C}}_{\text {top }}$ ) and moreover, is contained in a 3-manifold smoothly (resp. topologically) homology cobordant to $S^{3}$.

Proof To see the first claim, suppose that $E(P)^{-1}(\Psi(K))$ is equal in $\widehat{\mathcal{C}}_{*}$ to $\Psi(J)$ for some $J \in \mathcal{C}_{*}$ (for $*=$ ex or top) then $\Psi(K)=E(P)(\Psi(J))$. Then by the diagrams in (2-1), since $\Psi$ is injective, $K=P(J)$ and therefore, $K \in \operatorname{Im}\left(P: \mathcal{C}_{*} \rightarrow \mathcal{C}_{*}\right)$. The second statement follows from the following lemma since $\Psi(K)=\left(K, S^{3}\right)$.

Lemma 4.4 If $P \in \mathcal{S}_{\text {str }}$ and $(K, Y) \in \widehat{\mathcal{C}}_{\text {ex }}$ (resp. $\widehat{\mathcal{C}}_{\text {top }}$ ), then $E(P)^{-1}(K, Y)$ is a knot in a 3-manifold which is smoothly (resp. topologically) homology cobordant to $Y$.

Proof By definition, $E(P)(K, Y)$ is a knot in the 3-manifold

$$
\bar{Y}=S^{1} \times D^{2} \underset{\partial D^{2} \sim m(P)}{\cup} E(P) \underset{\substack{m(V) \sim m(K) \\ \ell(V) \sim \ell(K)}}{\cup} Y-K .
$$

But $S^{1} \times D^{2} \cup E(P)$ is just a solid torus with meridian $m(V)$ and therefore, these gluing instructions cut a solid torus out of $Y$ and then glue it back in the same way. Therefore, $\bar{Y}$ is diffeomorphic to $Y$ and $E(P)(K, Y)$ is a knot in $Y$. 
Let $E(P)^{-1}(K, Y)=\left(K^{\prime}, Y^{\prime}\right)$ and $E(P)\left(K^{\prime}, Y^{\prime}\right)=\left(K^{\prime \prime}, Y^{\prime \prime}\right)$. By the preceding paragraph, $Y^{\prime \prime}$ is diffeomorphic to $Y^{\prime}$. Since $E(P) \circ E(P)^{-1}$ is the identity map on $\widehat{\mathcal{C}}_{\text {ex }}$ (resp. $\left.\widehat{\mathcal{C}}_{\text {top }}\right), K$ is concordant to $K^{\prime \prime}$ in a smooth (resp. topological) homology cobordism between $Y$ and $Y^{\prime \prime}$, and hence $Y$ is smoothly (resp. topologically) homologically cobordant to $Y^{\prime \prime}=Y^{\prime}$. Since $E(P)^{-1}(K, Y)=\left(K^{\prime}, Y^{\prime}\right)$, the proof is completed.

The above proposition shows that if the satellite operator induced by a strong winding number \pm 1 pattern $P$ fails to be surjective on $\mathcal{C}_{\text {ex }}$ (resp. $\mathcal{C}_{\text {top }}$ ), ie there is some $K \in \mathcal{C}_{\text {ex }}$ (resp. $\mathcal{C}_{\text {top }}$ ) such that $K \neq P(J)$ for all knots $J$, then there exists a knot $K^{\prime}$ in a 3-manifold $Y^{\prime}$ smoothly (resp. topologically) homology cobordant to $S^{3}$, such that $\left(K^{\prime}, Y^{\prime}\right)$ is not exotically (resp. topologically) concordant to any knot in $S^{3}$, where $\left(K^{\prime}, Y^{\prime}\right)=E(P)^{-1}\left(K, S^{3}\right)$.

Acknowledgements The authors would like to thank Tim Cochran for many helpful conversations throughout this project, during part of which he was the doctoral advisor of the second author. The second author was partially supported by NSF-DMS-1309081.

\section{References}

[1] T Abe, I D Jong, Y Omae, M Takeuchi, Annulus twist and diffeomorphic 4-manifolds, Math. Proc. Cambridge Philos. Soc. 155 (2013) 219-235

[2] S Akbulut, A fake compact contractible 4-manifold, J. Differential Geom. 33 (1991) 335-356 MR1094459

[3] S Akbulut, R Kirby, Mazur manifolds, Michigan Math. J. 26 (1979) 259-284 MR544597

[4] S Akbulut, K Yasui, Corks, plugs and exotic structures, J. Gökova Geom. Topol. 2 (2008) 40-82 MR2466001

[5] T D Cochran, C W Davis, A Ray, Injectivity of satellite operators in knot concordance, J. Topol. 7 (2014) 948-964

[6] T D Cochran, B D Franklin, M Hedden, P D Horn, Knot concordance and homology cobordism, Proc. Amer. Math. Soc. 141 (2013) 2193-2208

[7] T D Cochran, S Harvey, C Leidy, Primary decomposition and the fractal nature of knot concordance, Math. Ann. 351 (2011) 443-508 MR2836668

[8] T D Cochran, K E Orr, P Teichner, Structure in the classical knot concordance group, Comment. Math. Helv. 79 (2004) 105-123 MR2031301

[9] M H Freedman, The topology of four-dimensional manifolds, J. Differential Geom. 17 (1982) 357-453 MR679066 
[10] S L Harvey, Homology cobordism invariants and the Cochran-Orr-Teichner filtration of the link concordance group, Geom. Topol. 12 (2008) 387-430 MR2390349

[11] M Hedden, P Kirk, Instantons, concordance, and Whitehead doubling, J. Differential Geom. 91 (2012) 281-319

[12] J Hempel, 3-manifolds, Ann. of Math. Studies 86, Princeton Univ. Press (1976) MR0415619

[13] R Kirby (editor), Problems in low-dimensional topology, AMS/IP Stud. Adv. Math. 2, Amer. Math. Soc. (1997)

[14] A S Levine, Non-surjective satellite operators and piecewise-linear concordance, preprint (2014) arXiv: 1405.1125

[15] J Levine, Homology cylinders: an enlargement of the mapping class group, Algebr. Geom. Topol. 1 (2001) 243-270 MR1823501

[16] RA Litherland, Signatures of iterated torus knots, from: "Topology of lowdimensional manifolds (Proceedings Second Sussex Conference)", (R A Fenn, editor), Lecture Notes in Mathematics 722, Springer, Berlin (1979) 71-84 MR547456

[17] C Livingston, P Melvin, Abelian invariants of satellite knots, from: "Geometry and topology", (J Alexander, J Harer, editors), Lecture Notes in Math. 1167, Springer, Berlin (1985) 217-227 MR827271

[18] D Rolfsen, Knots and links, Mathematics Lecture Series 7, Publish or Perish, Houston, TX (1990) MR1277811

[19] H Schubert, Über eine numerische Knoteninvariante, Math. Z. 61 (1954) 245-288 MR0072483

Department of Mathematics, The University of Wisconsin at Eau Claire 533 Hibbard Humanities Hall, Eau Claire, WI 54702, USA

Department of Mathematics, Brandeis University MS-050, 415 South St, Waltham, MA 02453, USA

daviscw@uwec.edu, aruray@brandeis.edu http://people.uwec.edu/daviscw/, http://people.brandeis.edu/ aruray/

Received: 24 October $2013 \quad$ Revised: 23 June 2015 
